\title{
Critical illness neuropathy in severe COVID-19: a case series
}

\author{
Tommaso Bocci ${ }^{1,2}$ - Laura Campiglio ${ }^{2}$. Manuela Zardoni ${ }^{2}$. Stefano Botta ${ }^{2}$ - Silvia Coppola ${ }^{3,4}$. Elisabetta Groppo ${ }^{2}$. \\ Davide Chiumello ${ }^{3,4} \cdot$ Alberto Priori $^{1,2,5}$
}

Received: 30 April 2021 / Accepted: 7 July 2021 / Published online: 3 September 2021

(C) Fondazione Società Italiana di Neurologia 2021

\begin{abstract}
Introduction Neurological complications of SARS-CoV-2 disease have received growing attention, but only few studies have described to date clinical and neurophysiological findings in COVID patients during their stay in intensive care units (ICUs). Here, we neurophysiologically assessed the presence of either critical illness neuropathy (CIP) or myopathy (CIM) in ICU patients.

Materials and Methods Patients underwent a neurophysiological assessment, including bilateral examination of the median, ulnar, deep peroneal and tibial motor nerves and of the median, ulnar, radial and sural sensory nerves. Needle electromyography (EMG) was performed for both distal and proximal muscles of the lower and upper limbs. In order to differentiate CIP from CIM, Direct Muscle Stimulation (DMS) was applied either to the deltoid or tibialis anterior muscles. Peak to peak amplitudes and onset latencies of the responses evoked by DMS (DMS ${ }_{\mathrm{amp}}, \mathrm{DMS}_{\mathrm{lat}}$ ) or by motor nerve stimulation (MNS $\mathrm{amp}_{\mathrm{a}}$, $\mathrm{MNS}_{\text {lat }}$ ) were compared. The ratio $\mathrm{MNS}_{\mathrm{amp}}$ to $\mathrm{DMS}_{\mathrm{amp}}(\mathrm{NMR})$ and the $\mathrm{MNS}_{\text {lat }}$ to $\mathrm{DMS}_{\text {lat }}$ difference (NMD: $\mathrm{MNS}_{\text {lat }}-\mathrm{DMS}_{\text {lat }}$ ) were also evaluated.

Results Nerve conduction studies showed a sensory-motor polyneuropathy with axonal neurogenic pattern, as confirmed by needle EMG. Both $\mathrm{MNS}_{\mathrm{amp}}$ and NMR were significantly reduced when compared to controls $(p<0.0001)$, whereas $\mathrm{MNS}_{\text {lat }}$ and NMD were markedly increased $(p=0.0049)$.

Conclusions We have described COVID patients in the ICU with critical illness neuropathy (CIP). COVID-related CIP could have implications for the functional recovery and rehabilitation strategies.
\end{abstract}

Keywords COVID-19 $\cdot$ SARS-CoV-2 $\cdot$ Neurophysiology $\cdot$ Critical illness neuropathy $\cdot$ Critical illness myopathy

Alberto Priori

alberto.priori@unimi.it

1 Aldo Ravelli" Center for Neurotechnology and Experimental Brain Therapeutics, Department of Health Sciences, University of Milan, Milan, Italy

2 Clinical Neurology Unit, ASST Santi Paolo \& Carlo, Milan, Italy

3 Intensive Care, Anesthesia and Resuscitation Unit, ASST Santi Paolo \& Carlo, Milan, Italy

4 Department of Health Sciences, University of Milan, Milan, Italy

5 Struttura Complessa Di Neurologia I, Ospedale Universitario San Paolo, Via Antonio di Rudinì 8, 20142 Milan, Italy

\section{Introduction}

SARS-Cov-2 disease has emerged in the past few months as the deadliest pandemic of the century, infecting over than $180,000,000$ people and causing about 3.9 million deaths worldwide (https://covid19.who.int). Neurological complications include large vessel strokes, acute polyradiculoneuropathies and epileptic seizures at onset, probably also accounting for a neurogenic component of the respiratory failure, as recently suggested by converging clinical, neurophysiological and histopathological evidence showing a direct viral invasion of the brainstem respiratory centers [1-5]. Nonetheless, only a few studies have described critical illness neuropathy or myopathy (CIP/CIM) in COVID patients during their stay in the intensive care units (ICUs). CIP/CIM may differently impact on the recovery and rehabilitation strategies, possibly delaying ICU discharge. Here, 
we neurophysiologically evaluated eight COVID patients in ICUs (seven males, mean age $56.0 \pm 9.3$ years).

\section{Materials and methods}

Patients with a Medical Research Council (MRC) score of less than 48/60 were included; each patient was ventilated for at least 1 week before the enrolment and had severe weakness on awakening; in four clinical signs of flaccid quadriparesis with arreflexia was found. Exclusion criteria were a pre-existing neuromuscular disease, a neuromuscular transmission failure, polyradiculoneuropathies and central nervous system disorders. None suffered from diabetes before the admission. At the time of neurophysiological assessment, each patient was awake and intubated (mean duration of intubation $10.2 \pm 4.1$ days); in the 60 min before none of them consumed drugs interfering with neuromuscular transmission. The mean time of hospitalization was $19.4 \pm 5.8$ days and the stay in ICU was $17.2 \pm 4.1$ days. During the hospitalization, three patients died because of COVID-related respiratory failure.

Each patient underwent nerve conduction studies, including bilateral examination of the median, ulnar, deep peroneal (from the extensor digitorum brevis muscle, EDB) and tibial (abductor hallucis brevis, AH) motor nerves (Compound Motor Action Potentials, CMAPs) and of the median, ulnar, radial and sural sensory nerves (Sensory Action Potentials, SAPs; Table 1); abductor digiti minimi muscle (ADM) responses to $3 \mathrm{~Hz}$ repetitive stimulation of the ulnar nerve were performed to exclude a neuromuscular transmission disease. To avoid the possibility of an acute polyradiculoneuropathy, both the "Sural-Radial Amplitude Ratio" (SRAR) and the "Sensory Ratio" (SR) were calculated (Table 2) [6]. Distal and proximal muscles were assessed, using disposable concentric needles (tibialis anterior, TA, and vastus medialis, VM; first digital interosseus, FDI, and deltoid); the number of insertions was two for each muscle and rest activity was monitored for 60 ".

Following the conventional assessment, Direct Muscle Stimulation (DMS) was applied to the tibialis anterior muscle in each patient and also in the deltoid muscle in three patients. The methods for DMS is reported in detail elsewhere [7, 8]; briefly, a monopolar needle electrode (length $37 \mathrm{~mm}$; diameter $0.36 \mathrm{~mm}$; stimulating surface $0.28 \mathrm{~mm}^{2}$ ) served as cathode and was inserted in the distal third part of the muscle, while a subdermal needle electrode (length $12 \mathrm{~mm}$ ) was placed $10 \mathrm{~mm}$ laterally. The muscle was stimulated at different depths at increasing strength, until a twitch was evoked (from 10.0 to $100 \mathrm{~mA} ; 0.1 \mathrm{~ms}$ and $0.5 \mathrm{~Hz}$ ); then, a concentric needle was inserted $15 \mathrm{~mm}$ proximally to the cathode, at increasing depths until a response of maximum amplitude was recorded (band-pass $20 \mathrm{~Hz}-10 \mathrm{kHz}$ ) [7].
Any response with an onset latency shorter than $2.0 \mathrm{~ms}$ was excluded, because likely obtained by nerve-ending stimulation. Finally, the axillary or deep peroneal nerves were stimulated with surface electrodes (motor nerve stimulation, MNS). Peak to peak amplitudes and onset latencies of the responses evoked by DMS (DMS ${ }_{\mathrm{amp}}, \mathrm{DMS}_{\text {lat }}$ ) or by MNS $\left(\mathrm{MNS}_{\mathrm{amp}}, \mathrm{MNS}_{\text {lat }}\right)$ were measured. The ratio $\mathrm{MNS}_{\mathrm{amp}}$ to $\mathrm{DMS}_{\mathrm{amp}}(\mathrm{NMR})$ and the $\mathrm{MNS}_{\text {lat }}$ to $\mathrm{DMS}_{\text {lat }}$ difference (NMD: $\mathrm{MNS}_{\text {lat }}-\mathrm{DMS}_{\mathrm{lat}}$ ) were assessed.

The whole electrophysiological protocol required about $1 \mathrm{~h}$ for each patient.

Normative data were acquired in eight healthy subjects (six males, mean age $52.1 \pm 10.9$ years). Statistical analysis was performed using a Wilcoxon test $(p<0.05)$.

\section{Results}

Neurophysiological findings are reported in Tables 1 and 2; traces from a representative patient are provided in Fig. 1. Normative data are in line with those reported elsewhere [7].

CMAPs showed reduced amplitudes, especially at the lower limbs, with onset latencies within normal limits; $\mathrm{F}$-waves were not recordable from the $\mathrm{AH}$ muscle in three patients and had an increased onset latency in the remaining cases, with a persistence lower than 50\% (AH: $65.1 \pm 6.7 \mathrm{~ms}$; ADM: $37.2 \pm 5.3 \mathrm{~ms})$. SAP $_{\mathrm{amp}}$ were significantly impaired and in four cases SAPs from the sural nerve were not detectable.

Concentric needle electromyography revealed a neurogenic pattern, with denervation activity, represented by positive sharp waves and in two cases by fibrillation potentials.

$\mathrm{MNS}_{\mathrm{amp}}$ and NMR were significantly reduced in patients (NMR for the tibial muscle: $p<0.0001$, Fig. 1, Table 2) while $\mathrm{MNS}_{\text {lat }}$ and NMD were increased (NMD: $p=0.0049$, Fig. 1, Table 2).

\section{Discussion}

Our results suggest a predominant critical illness neuropathy (CIP), in line with some recent papers about intensive care unit acquired weakness in severe COVID patients, and differ from those previously reported in the literature, showing a higher prevalence of myopathy in non-COVID patients with CIP/CIM syndromes [7, 9, 10].

DMS technique has been proposed for testing muscle fibers excitability, in order to understand the mechanisms of ICU acquired paresis, especially when voluntary activity is absent [7-9]. The involvement of the peripheral nervous system may significantly impact on the patient's prognosis, as CIP is characterized by a worst outcome than CIM, probably related to the associated multiple organ failure $[9,11]$. 


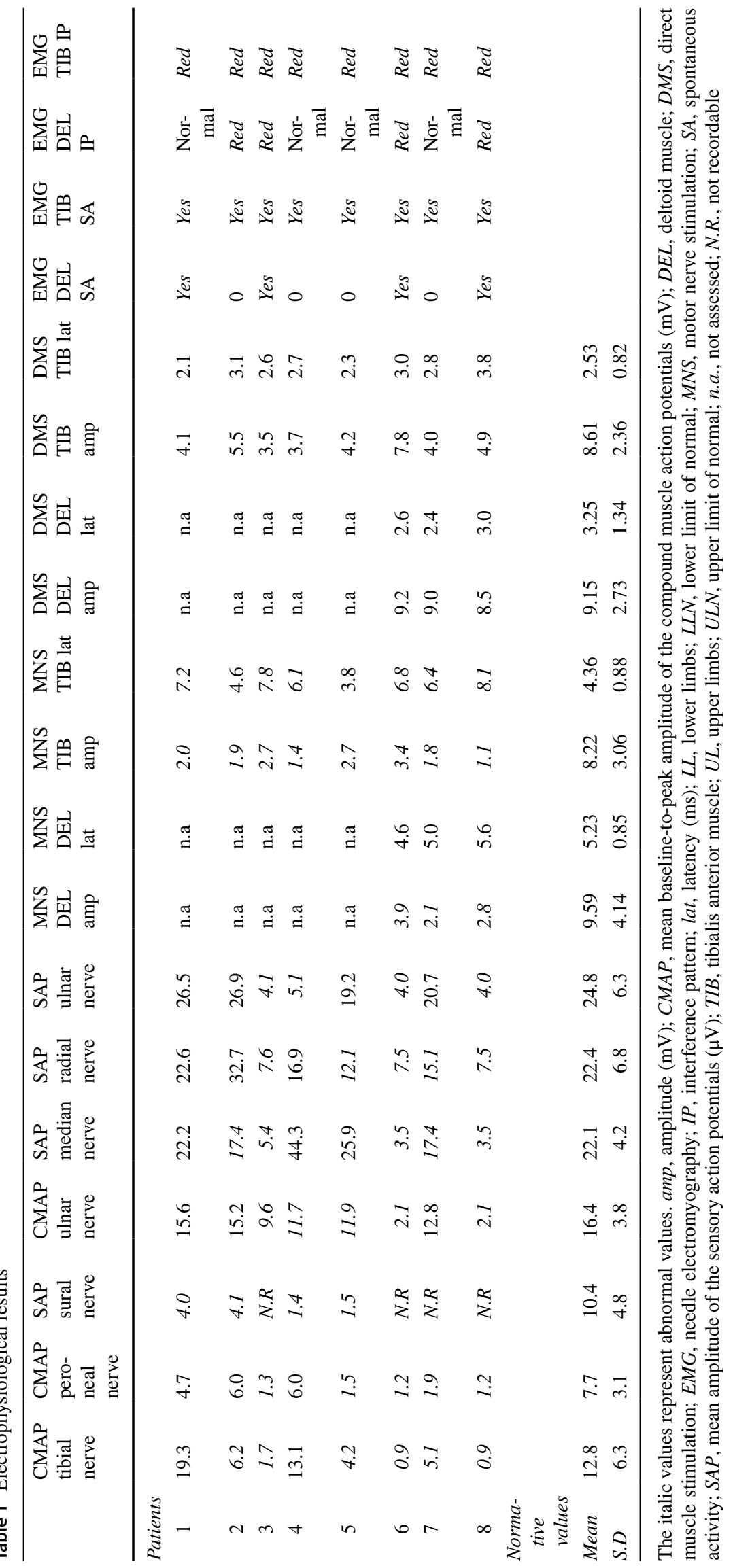


Table 2 Neurophysiological outcome (derived measures) and CK levels

\begin{tabular}{llllllll}
\hline & NMD DEL $(\mathrm{ms})$ & NMR DEL & NMD TIB (ms) & NMR TIB & SRAR & SR & CK levels (UI/L) \\
\hline Patients & & & & & & & \\
1 & n.a & n.a & 5.1 & 0.49 & 0.18 & 0.70 & N.V \\
2 & n.a & n.a & 1.5 & 0.35 & 0.13 & 1.03 & 435 UI/L \\
3 & n.a & n.a & 5.2 & 0.77 & n.e & n.e & 518 UI/L \\
4 & n.a & n.a & 3.4 & 0.38 & 0.09 & 0.33 & N.V \\
5 & n.a & n.a & 1.5 & 0.64 & 0.12 & 0.36 & N.V \\
6 & 2.0 & 0.42 & 3.8 & 0.44 & n.e & n.e & N.V \\
7 & 2.6 & 0.23 & 3.6 & 0.45 & n.e & n.e & N.V \\
8 & 2.9 & 0.33 & 4.3 & 0.22 & n.e & n.e & 341 UI/L \\
Normative values & & & & & & \\
Mean & 1.82 & 1.20 & 1.75 & 0.95 & & $>0.4^{* \dagger}$ & $<1.1^{*}$ \\
S.D & 0.59 & 0.21 & 0.55 & 0.20 & &
\end{tabular}

The italic values represent abnormal values. $N M D$, difference of response latency between motor nerve stimulation and direct muscle stimulation (ms); $N M R$, ratio of response amplitude from motor nerve stimulation to direct muscle stimulation; red, reduced; SRAR, "sural-radial amplitude ratio" $\left(\mathrm{SAP}_{\text {sural }} / \mathrm{SAP}_{\text {radial }}\right) ; S R$, "sensory ratio", as expressed by the formula $\left(\mathrm{SAP}_{\text {sural }}+\mathrm{SAP}_{\text {radial }}\right) /\left(\mathrm{SAP}_{\text {median }}+\mathrm{SAP}_{\text {ulnar }}\right)$; n.a., not assessed; n.e., not evaluable, because of the absence of $\mathrm{SAP}_{\text {sural }}$. $C K$, creatin-phospho-kinase; N.V., normal values (within normal range, defined as $<190 \mathrm{UI} / \mathrm{L}$ )

*Based on Al-Schekhlee et al. (2007)

${ }^{\dagger}$ SRAR values over 0.4 are for controls and patients with GBS, whereas in length-dependent, dying-back polineuropathies (e.g. CIP) SRAR is usually lower

A

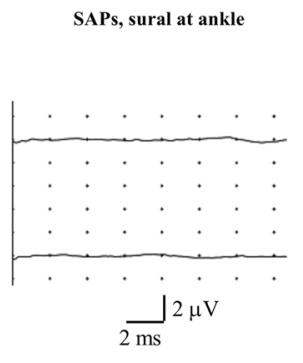

F-waves, ulnar at ADM

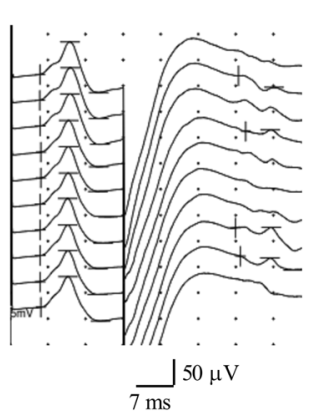

B
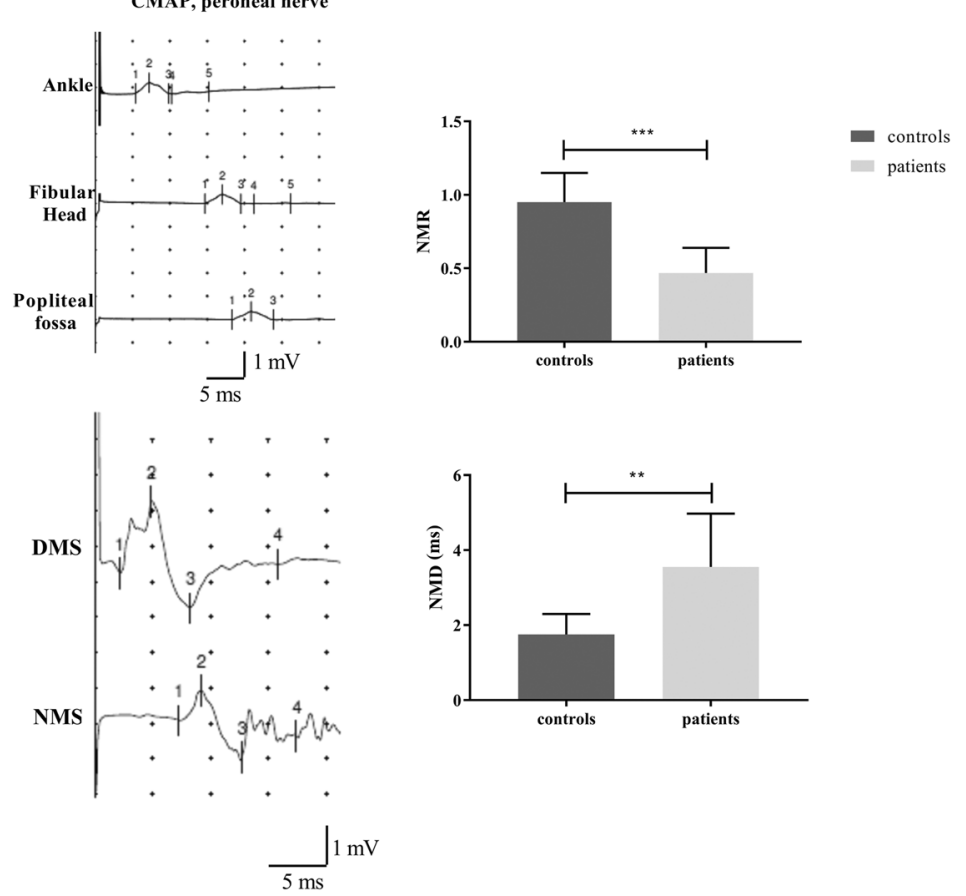

Fig. 1 Neurophysiological findings. The figure in A shows neurophysiological traces from a representative patient. Top: sensory action potentials (SAPs) from the right sural nerve (not recordable) and CMAP (reduced amplitude) derived from the right extensor digitorum brevis are shown; at the bottom (left) F-waves from the left ulnar nerve are provided (with abnormal onset latency, reduced amplitude and impaired representation). Bottom: (right), DMS (top trace) and NMS (bottom), derived from the tibial muscle, in a COVID patient (male, 52 years); note the amplitude reduction when NMS was compared to DMS; B Histograms showing NMR and NMD (ms) values, both in patients and controls (TA muscle, stimulation of the deep peroneal nerve; values are reported as mean \pm 1 standard error, S.E.); note the reduction of NMR, paralleled by a significant increase of NMD, suggesting a predominant neuropathy $(* * * p<0.001$; $* * p<0.01$ ) 
Interestingly, two studies have recently confirmed our findings supporting a higher prevalence of CIP in COVID19 compared with a non-COVID-19 cohort [12, 13]. Of note, Bax and colleagues also found a correlation between the impairment of neurographic parameters and plasma biomarkers of nerve injury, including Neurofilament Light chain (NfL) and Glial Fibrillary Acidic protein (GFAp) levels, thus suggesting CIP may be a consequence of COVID-19 itself rather than an effect of the severe illness [12]. Nonetheless, different from our data, none of these two studies used a non-conventional electrodiagnostic approach.

Previously, other papers had described single cases of critical illness myopathy in COVID patients $[14,15]$ and one assessed DMS and NMS parameters [15]. Another study described a larger case series, showing a predominant myogenic pattern [16]; the authors interestingly reported the histopathological findings from four muscle biopsies, but they did not use non-conventional electrodiagnostic approaches. Moreover, in the patient described by Tankisi and co-workers, a quantitative needle electromyography (Multiple Motor Unit Analysis, MMUA) was also performed.

The discrepancy between their results and ours may be due to the duration of hospitalization before EMG, longer than in our sample (until 2 months of hospitalization in ICU), different therapeutic approaches (e.g. steroids; hydroxychloroquine, not used in our sample) or concomitant bacterial infections $[15,17]$. Other confounding factors may be related to different ages and small sample sizes, due to critical conditions in which the neurophysiological evaluation was performed.

Finally, in none of the abovementioned papers, the differential diagnosis between CIP/CIM and GBS was assessed by using derived measures, as SR and SRAR. These parameters are known to have a higher diagnostic sensitivity when compared to the conventional electrodiagnostic assessment and the SR may substitute for "sural sparing" in technically difficult situations [6].

Although our findings do not fully reflect a multineuropathic pattern, showing a bilateral, symmetric and lengthdependent neuropathy, without any predominance of ulnar and deep peroneal nerve impairment, we cannot exclude the possibility, at least in part, of a vasculitic involvement of the peripheral nervous system. More important, we cannot rule out the possibility of a direct viral invasion of the peripheral nervous system, as likely occurs at the central level $[1,2]$. Probably, in the next months, a growing number of critical illness neuromyopathy will be described in COVID-19 survivors, in terms of both patients and case series, thus increasing long-term complications and healthcare costs, highlighting novel insights into the neuropathology of COVID-19.
Acknowledgements The authors are grateful to Mr. Denis Dreon for his excellent technical assistance.

Funding This study was supported by the donation of the "Romeo and Enrica Invernizzi" Foundation ("Bando COVID-19").

Data availability The corresponding author has full access to data and has the right to publish such data. Data will be available upon reasonable request to the corresponding author.

Code availability Not applicable.

\section{Declarations}

Ethical approval We confirm that we have read the journal's position on issues involved in ethical publication and affirm that this report is consistent with those guidelines.

Consent to participate The study was approved by the institutional review board and the ethics committee at "Azienda Socio-Sanitaria Territoriale Santi Paolo e Carlo, Milano, Italia". The study has been conducted in accordance with the ethical standards laid down in the Declaration of Helsinki.

Conflict of interest The authors declare no competing interests.

\section{References}

1. Moro E, Priori A, Beghi E, Helbok R, Campiglio L, Bassetti CL, Bianchi E, Maia LF, Ozturk S, Cavallieri F, Zedde M, Sellner J, Bereczki D, Rakusa M, Di Liberto G, Sauerbier A, Pisani A, Macerollo A, Soffietti R, Taba P, Crean M, Twardzik A, Oreja-Guevara C, Bodini B, Jenkins TM, von Oertzen TJ, Force EANcC-T (2020) The international European Academy of Neurology survey on neurological symptoms in patients with COVID-19 infection. Eur J Neurol 27(9):1727-1737. https://doi.org/10.1111/ene.14407

2. Bocci T, Bulfamante G, Campiglio L, Coppola S, Falleni M, Chiumello D, Priori A (2021) Brainstem clinical and neurophysiological involvement in COVID-19. J Neurol. https://doi.org/10. 1007/s00415-021-10474-0

3. Priori A, Baisi A, Banderali G, Biglioli F, Bulfamante G, Canevini MP, Cariati M, Carugo S, Cattaneo M, Cerri A, Chiumello D, Colosio C, Cozzolino M, D'Arminio Monforte A, Felisati G, Ferrari D, Gambini O, Gardinali M, Marconi AM, Olivari I, Orfeo NV, Opocher E, Pietrogrande L, Previtera A, Rossetti L, Vegni E, Toschi V, Zuin M, Centanni S (2020) The many faces of Covid19 at a glance: a university hospital multidisciplinary account from Milan. Italy Front Public Health 8:575029. https://doi.org/ 10.3389/fpubh.2020.575029

4. Bulfamante G, Bocci T, Falleni M, Campiglio L, Coppola S, Tosi D, Chiumello D, Priori A (2021) Brainstem neuropathology in two cases of COVID-19: SARS-CoV-2 trafficking between brain and lung. J Neurol. https://doi.org/10.1007/s00415-021-10604-8

5. Matschke J, Lutgehetmann M, Hagel C, Sperhake JP, Schroder AS, Edler C, Mushumba H, Fitzek A, Allweiss L, Dandri M, Dottermusch M, Heinemann A, Pfefferle S, Schwabenland M, Sumner Magruder D, Bonn S, Prinz M, Gerloff C, Puschel K, Krasemann S, Aepfelbacher M, Glatzel M (2020) Neuropathology of patients with COVID-19 in Germany: a post-mortem case series. Lancet Neurol 19(11):919-929. https://doi.org/10.1016/S1474-4422(20) 30308-2 
6. Al-Shekhlee A, Robinson J, Katirji B (2007) Sensory sparing patterns and the sensory ratio in acute inflammatory demyelinating polyneuropathy. Muscle Nerve 35(2):246-250. https://doi.org/10. 1002/mus. 20660

7. Lefaucheur JP, Nordine T, Rodriguez P, Brochard L (2006) Origin of ICU acquired paresis determined by direct muscle stimulation. J Neurol Neurosurg Psychiatry 77(4):500-506. https://doi.org/10. 1136/jnnp.2005.070813

8. Rich MM, Bird SJ, Raps EC, McCluskey LF, Teener JW (1997) Direct muscle stimulation in acute quadriplegic myopathy. Muscle Nerve 20(6):665-673. https://doi.org/10.1002/(sici)10974598(199706)20:6\%3c665::aid-mus2\%3e3.0.co;2-6

9. Trojaborg W, Weimer LH, Hays AP (2001) Electrophysiologic studies in critical illness associated weakness: myopathy or neuropathy-a reappraisal. Clin Neurophysiol 112(9):1586-1593. https://doi.org/10.1016/s1388-2457(01)00572-7

10. Bednarik J, Lukas Z, Vondracek P (2003) Critical illness polyneuromyopathy: the electrophysiological components of a complex entity. Intensive Care Med 29(9):1505-1514. https://doi.org/10. 1007/s00134-003-1858-0

11. Leijten FS, Harinck-de Weerd JE, Poortvliet DC, de Weerd AW (1995) The role of polyneuropathy in motor convalescence after prolonged mechanical ventilation. JAMA 274(15):1221-1225

12. Bax F, Lettieri C, Marini A, Pellitteri G, Surcinelli A, Valente M, Budai R, Patruno V, Gigli GL (2021) Clinical and neurophysiological characterization of muscular weakness in severe COVID-19. Neurol Sci 42(6):2173-2178. https://doi.org/10.1007/ s10072-021-05110-8

13. Frithiof R, Rostami E, Kumlien E, Virhammar J, Fallmar D, Hultstrom M, Lipcsey M, Ashton N, Blennow K, Zetterberg H,
Punga AR (2021) Critical illness polyneuropathy, myopathy and neuronal biomarkers in COVID-19 patients: a prospective study. Clin Neurophysiol 132(7):1733-1740. https://doi.org/10.1016/j. clinph.2021.03.016

14. Tankisi H, Tankisi A, Harbo T, Markvardsen LK, Andersen H, Pedersen TH (2020) Critical illness myopathy as a consequence of Covid-19 infection. Clin Neurophysiol 131(8):1931-1932. https:// doi.org/10.1016/j.clinph.2020.06.003

15. Bagnato S, Boccagni C, Marino G, Prestandrea C, D'Agostino T, Rubino F (2020) Critical illness myopathy after COVID-19. Int J Infect Dis 99:276-278. https://doi.org/10.1016/j.ijid.2020.07.072

16. Cabanes-Martinez L, Villadoniga M, Gonzalez-Rodriguez L, Araque L, Diaz-Cid A, Ruz-Caracuel I, Pian H, Sanchez-Alonso S, Fanjul S, Del Alamo M, Regidor I (2020) Neuromuscular involvement in COVID-19 critically ill patients. Clin Neurophysiol 131(12):2809-2816. https://doi.org/10.1016/j.clinph.2020.09. 017

17. Khan J, Harrison TB, Rich MM, Moss M (2006) Early development of critical illness myopathy and neuropathy in patients with severe sepsis. Neurology 67(8):1421-1425. https://doi.org/10. 1212/01.wnl.0000239826.63523.8e

Publisher's note Springer Nature remains neutral with regard to jurisdictional claims in published maps and institutional affiliations. 